\title{
基于壳聚糖物理网络的高强韧双网络水凝胶的构建、调控与应用
}

\author{
杨艳宇 $a, b \quad$ 王星 $*, b$ 吴德成*,b,c \\ ( ${ }^{a}$ 郑州大学材料科学与工程学院 郑州 450001) \\ $\left({ }^{b}\right.$ 中国科学院化学研究所 北京 100190) \\ ( ${ }^{c}$ 南方科技大学生物医学工程系 深圳 518055)
}

\begin{abstract}
摘要 双网络水凝胶由两个具有相反物理性质的交联网络构成, 硬而脆的第一网络在变形过程中断裂耗散能量, 从而 增㓞凝胶. 当第一网络为具有重建能力的物理网络时, 双网络水凝胶表现出优异的抗软化和机械稳定性. 目前双网络 水凝胶第一物理网络类型单一、结构和力学调控繁琐, 因而其开发和应用受到限制. 针对上述问题, 作者发展了硬而脆 的壳聚糖物理网络构建的新策略, 壳聚糖的网络类型、结构和物理性质均能简便调控, 然后将其作为第一网络成功地 制备了多种高强韧且结构性能可灵活调控的双网络水凝胶, 在抗冻敷料、生物医用、柔性电子和可穿戴设备等领域具 有重要的应用价值. 构建壳聚糖基双网络水凝胶的策略操作简单、普适通用, 能够有力促进高强韧水凝胶的开发、功 能化及应用。
\end{abstract}

关键词＼cjkstart壳聚糖物理网络; 高强韧水凝胶; 结构和性能调控; 耐冻性; 柔性可穿戴设备

\section{Chitosan-Based High-Mechanical Double-Network Hydrogels: Construction, Modulation and Applications}

\author{
Yang, Yanyu $^{a, b} \quad$ Wang, Xing ${ }^{*, b} \quad$ Wu, Decheng ${ }^{*, b, c}$ \\ ( ${ }^{a}$ College of Materials Science and Engineering, Zhengzhou University, Zhengzhou 450001, China) \\ $\left({ }^{b}\right.$ Institute of Chemistry, Chinese Academy of Sciences, Beijing 100190, China) \\ ( ${ }^{c}$ Department of Biomedical Engineering, Southern University of Science and Technology, Shenzhen 518055, China)
}

\begin{abstract}
Double-network (DN) hydrogels are composed of two asymmetric networks with contrasting properties, wherein the rigid and brittle network serving as sacrificial bonds effectively dissipates energy to enhance the mechanical performance. The first reconstructable physical network endows the DN hydrogels with outstanding anti-soften and mechanical stability. However, the monotonous type of physical networks and the difficulty in tailoring structure and mechanics greatly limit the development and application of DN hydrogels. Focusing on these problems, we have fabricated the rigid and brittle chitosan physical network with adjustable network type, structure and property and further constructed various chitosan-based DN hydrogels with high mechanical performance and tunable mechanics. The hydrogels were potential materials for anti-freezing dresses, biomedical materials, flexible electronics and wearable devices. The universal strategy of constructing chitosan-based DN hydrogels was beneficial for developing various functional and high-mechanical hydrogels and broadening their applications.

Keywords chitosan physical network; strong and tough hydrogel; structural and mechanical regulation; freezing tolerance; flexible wearable device
\end{abstract}

\section{1 引言}

双网络水凝胶是一类典型的高强韧水凝胶, 由两个 具有相反物理性质的交联网络组成, 第一网络具有硬而 脆的特点, 第二网络具有柔软和优异的延展性, 两个网 络能够协同提高水凝胶的机械性能 ${ }^{[1]}$. 双网络水凝胶的 增韧机理为: 第一网络在凝胶变形的过程中充当 “牺牲 键” 发生断裂发挥耗散能量的作用, 断裂形成的小碎块 在第二网络中发挥 “交联点” 的作用, 第二网络在小形 变时维持凝胶的完整性并贡献水凝胶的延展性. 根据所
构成的网络类型, 双网络水凝胶分为: 化学-化学、物 理-化学和物理-物理双网络水凝胶. 物理-化学和物理物理双网络水凝胶的第一网络是可逆物理网络, 断裂之 后能够修复, 赋予水凝胶优异的自恢复和抗软化性, 这 在反复受力的承重软组织和柔性电子设备领域是非常 必要且亟需的 ${ }^{[2]}$.

2008 年, 锁志刚团队 ${ }^{[3]}$ 采用一锅法制备了 $\mathrm{Ca}^{2+}$-海 藻酸盐-聚丙烯酰胺离子-共价混合双网络水凝胶, 表现 出优异的延展性(2000\%)、高断裂能(约 $9000 \mathrm{~J} \bullet \mathrm{m}^{-2}$ )、缺

\footnotetext{
*E-mail: wangxing@iccas.ac.cn; dcwu@iccas.ac.cn

Received August 16, 2020; published September 15, 2020.

Project supported by the National Natural Science Foundation of China (Nos. 51803188, 51973226, 21725403).

项目受国家自然科学基金(Nos. 51803188, 51973226, 21725403)资助.
} 
口不敏感性和自恢复性能. 在此基础上, 结冷胶、卡拉 胶和黄原胶等酸性多糖的离子网络被开发用于可逆第 一网络, 构建了多种抗软化的双网络水凝胶 ${ }^{[4-6]}$. 此后, 琼脂氢键网络和聚乙烯醇微晶网络也作为硬而脆的第 一网络用于制备物理-化学和双物理网络水凝胶 ${ }^{[2,7-8]}$. 尽管酸性多糖(海藻酸钠、结冷胶、卡拉胶和黄原胶)、 琼脂、聚乙烯醇均能够用于构建硬而脆的第一网络, 但 是每一种原料仅能制备单一类型的物理网络(离子、氢 键或微晶网络). 此外, 水凝胶机械性能的调控费时耗 力, 需要回溯到制备初期通过改变各成分的含量、交联 剂的用量及两个网络间的比例等来实现. 物理网络的单 一性及机械性能调节的繁琐性极大地限制了抗软化双 网络水凝胶的拓展和应用. 基于上述考虑, 我们思考能 否找到一种类型、结构和性能均可调控的物理网络用于 构建抗软化的双网络水凝胶, 以获得种类丰富的双网络 水凝胶并实现其微观结构和机械性能的灵活调控?

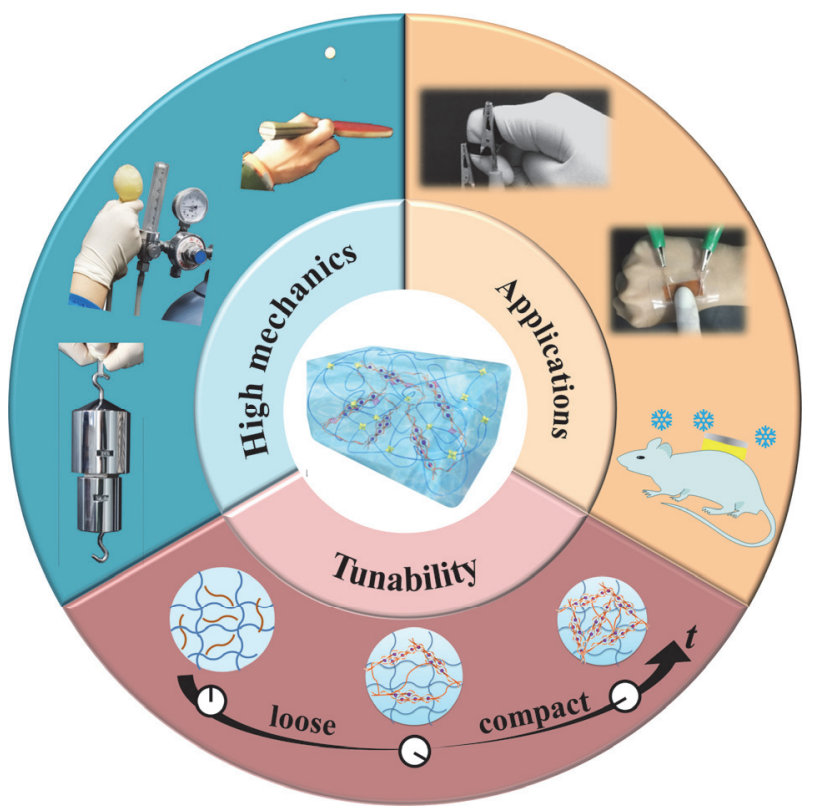

图 1 壳聚糖基双网络水凝胶的高机械性能、结构调控性及应用

Figure 1 The high mechanical performance, tunable structure and applications of chitosan-based DN hydrogel

壳聚糖在碱性或者盐溶液中能够形成结晶、链缠结 或离子网络 ${ }^{[9-11]}$. 然而壳聚糖的低溶解度和溶液的高粘 度导致生成的壳聚糖物理网络脆而弱, 无法满足双网络 水凝胶第一网络硬而脆的物理需求, 因而壳聚糖物理网 络很难应用到双网络水凝胶的构建中. 本课题组开发了 一种将短链壳聚糖复合水凝胶通过一步浸泡法构建高 强韧双网络水凝胶的方法, 成功地将壳聚糖结晶、链缠 结和离子物理网络引入到双网络水凝胶中, 构建了多种 壳聚糖基双网络水凝胶 ${ }^{[12]}$. 我们系统地研究了壳聚糖 物理网络的成胶机理, 通过控制复合水凝胶浸泡溶液的 种类和浸泡时间灵活调控双网络水凝胶的类型、微观结 构和机械性能, 进而探索了水凝胶在防冻敷料、柔性电
子和可穿戴设备等领域的应用(图 1).

\section{2 壳聚糖物理网络的形成机理}

壳聚糖作为自然界唯一的碱性多糖，化学名为 $(1,4)$-2-乙酰氨基-2-脱氧- $\beta$ - $D$ - 葡聚糖，由 $N$-乙酰- $D$-葡萄 糖胺和 $N$-葡萄糖胺两种重复单元通过 $\beta-(1-4)$ 糖苷键连 接组成(图 2). 壳聚糖具有良好的生物相容性和可降解 性, 已经广泛应用于药物释放系统、组织工程、皮肤修 复材料等领域 ${ }^{[13-14]}$. 壳聚糖物理网络的形成需要满足两 个条件：(1)壳聚糖分子内或者分子间的作用足够强，能 够在网络中形成半渗透性质的物理结合点; (2)物理网络 具备一定的保水性 ${ }^{[11]}$.

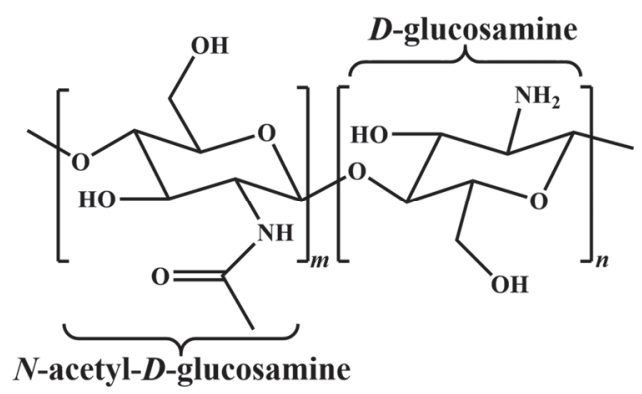

图 2 壳聚糖的结构式

Figure 2 Structure of chitosan chain

\section{1 壳聚糖结晶网络}

在碱性环境中，壳聚糖分子链上的氨基去质子化， 分子间的离子排斥作用减弱, 分子链的疏水性增强, 分 子链间的氢键作用力增强, 诱导壳聚糖分子形成微晶结 构, 此微晶结构作为结晶网络的物理结合点 ${ }^{[9,11]}$. 2008 年 Domard 等 ${ }^{[9]}$ 报道了用氢氧化钠 $(\mathrm{NaOH})$ 和水替换的方 法制备洋忽状的壳聚糖结晶网络凝胶. 首先将乙醇加入 壳聚糖的酸性溶液中制备壳聚糖的水醇凝胶，之后置于 $1 \mathrm{~mol} \cdot \mathrm{L}^{-1} \mathrm{NaOH}$ 溶液中形成壳聚糖结晶网络凝胶 ${ }^{[15]}$; 然后将 $\mathrm{NaOH}$ 溶液更换为水中止壳聚糖的成胶过程; 经 过多次更换 $\mathrm{NaOH}$ 和水成功地制备得到多层结构的壳 聚糖凝胶薄膜(图 3).

\section{2 壳聚糖链缠结网络}

当饱和的氯化钠溶液加入壳聚糖的溶液中，强烈的 盐析作用导致壳聚糖分子间的静电排斥力减小, 疏水作 用力增强, 促使壳聚糖分子链聚集而产生物理缠结, 此 缠结点能够作为链缠结网络的物理结合点 ${ }^{[9-10]}$. 此外, 其它的高溶解度卤盐也会诱发壳聚糖链缠结网络的形 成, 如氯化钙、氯化钾和氯化镁等. 然而壳聚糖链缠结 网络在水中会逐渐瓦解, 致使固体凝胶转变为溶胶.

\section{3 壳聚糖离子网络}

在加入多价阴离子盐(如硫酸根、磷酸根和柠檬酸 根)或多价阴离子分子时，强烈的盐析作用有助于壳聚 糖分子去水化，分子间的疏水作用和氢键作用增强， $N$ - 


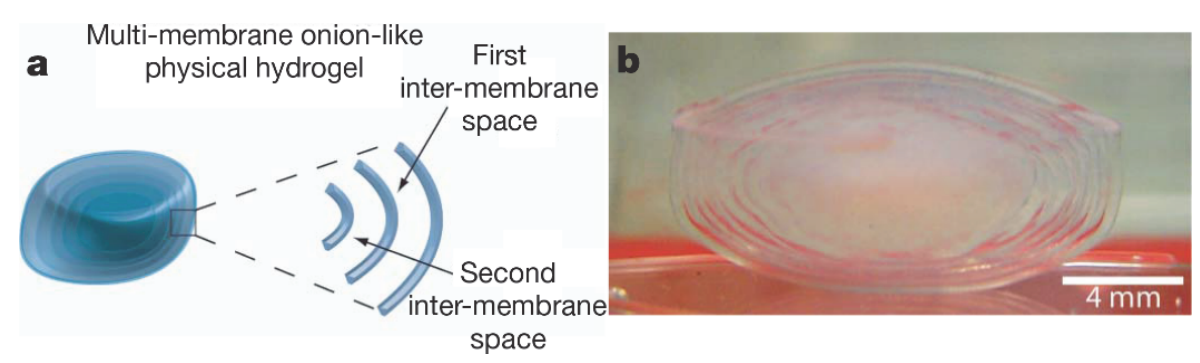

图 3 多层结构的壳聚糖结晶网络凝胶 ${ }^{[9]}$

Figure 3 Multi-membrane chitosan crystal network hydrogel ${ }^{[9]}$

葡萄糖胺能够和多价阴离子形成 “二叉” 和 “多叉” 配 位键, 从而形成以离子配位键为物理结合点的离子交联 网络 ${ }^{[11,16-17]}$ (图 4). 壳聚糖离子网络的性质依赖于阴离子 试剂的尺寸和电荷密度. 分子量小的阴离子试剂具有良 好的渗透性, 能够快速地进入壳聚糖基质中形成交联网 络; 高电荷密度的阴离子试剂能够和壳聚糖形成紧密而 牢固的离子配位作用 ${ }^{[17]}$.
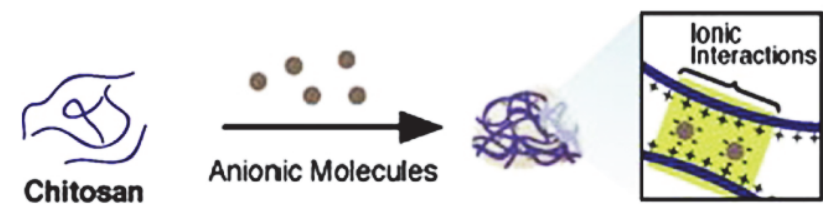

图 4 壳聚糖离子网络凝胶 ${ }^{[11]}$

Figure 4 Scheme of chitosan ionic network hydrogel ${ }^{[11]}$

此外, 壳聚糖与负电性的聚电解质能够通过静电作 用形成聚电解质复合作用物理网络, 该作用力比分子间 的二级作用(氢键作用、范德华力等)强 ${ }^{[18]}$. 壳聚糖-聚电 解质复合作用凝胶制备过程简单, 生物安全性良好, 但 是该物理网络表现出软而弱的特点, 无法作为第一网络 用于构建双网络水凝胶.

\section{3 壳聚糖基双网络水凝胶的构建}

尽管壳聚糖能够在不同类型的盐溶液中形成多种 物理网络, 但是长链壳聚糖在制备双网络水凝胶时存在 两个明显的困难: (1)溶解度差(低于 $3 \%$ ), 生成的壳聚糖 物理网络固含量低、脆而弱, 无法满足双网络水凝胶第 一网络硬而脆的需求; (2)溶液粘度高, 难于与其它材料 均匀混合. 这两个缺点导致壳聚糖物理网络未能应用于 双网络凝胶的构建. 针对上述问题, 我们采用高溶解度 的短链壳聚糖(脱乙酰度 $>90 \%$, 分子量约为 $10 \mathrm{kDa}$, $1 \%(w / V)$ 溶液的粘度为 $45 \mathrm{mPa} \cdot \mathrm{s})$ 并发挥其易于构建多种 类型物理网络的优势来制备壳聚糖基双网络水凝胶, 首 先将较高浓度(质量分数大于 $5 \%$ ) 的短链壳聚糖通过氢 键作用力均匀分散于另一柔性网络中制备得到壳聚糖 基复合水凝胶, 然后使用不同种类的盐溶液浸泡策略分 别构建三类硬而脆的壳聚糖物理网络, 从而得到多种壳 聚糖基高强韧双网络水凝胶. 这种方法具有以下优势: (1)操作简单、一步浸泡法快速构建硬而脆的壳聚糖物理
网络, 实现壳聚糖基复合水凝胶向高强韧双网络水凝胶 的快速转变; (2)仅仅改变浸泡溶液的类型能够灵活改变 壳聚糖物理网络类型; (3)调节浸泡时间能够实现双网络 水凝胶微观结构和机械性能的灵活调控; (4)壳聚糖物理 网络的解交联或者解离能够有效地耗散能量, 提升凝胶 的韧性; (5)壳聚糖物理网络的重建能力赋予凝胶快速的 自恢复性和抗疲劳性. 该制备策略丰富了高强韧水凝胶 的设计思路, 有利于扩大双网络水凝胶的应用范围.

\section{1 微晶-共价双网络水凝胶}

如图 5 所示, 首先将壳聚糖 $(\mathrm{CS})$ 与经典的聚丙烯酰 胺(PAM)共价网络通过氢键作用制备成为壳聚糖复合水 凝胶, 然后将其浸泡于 $1 \mathrm{~mol} \cdot \mathrm{L}^{-1} \mathrm{NaOH}$ 中, 壳聚糖分子 链上的氨基去质子化，分子的疏水性增强，分子链间的 氢键作用力增强, 诱导壳聚糖分子形成微晶交联网 络 ${ }^{[9,11,15]}$, 得到 CS-PAM 微晶-共价双网络水凝胶. 硬而 脆的壳聚糖微晶网络和柔软且易于延展的聚丙烯酰胺 网络能够协同提高水凝胶的机械性能(拉伸强度: 2.12 $\mathrm{MPa}$, 弹性模量: $318.7 \mathrm{kPa}$, 断裂韧性高达 $12.9 \mathrm{~kJ} \cdot \mathrm{m}^{-2}$ ). 水凝胶具有优异的负重能力和抗碾压性能, 在汽车的反 复碾压实验中依然能够保持结构完整(图 6A, 6B). 在变 形的过程中，壳聚糖的微晶网络充当 “牺牲键” 发生断 裂并耗散能量, 聚丙烯酰胺共价网络发挥裂纹桥联的作 用以稳定凝胶的变形行为, 均匀的小孔结构传递缺口处 的应力, 避免应力集中, 共同抑制缺口的增长. 当具有 缺口的水凝胶断裂应力与模量的比值 $\left(\sigma_{\mathrm{f}} / E\right)$ 大于 2 时, 会 出现缺口不敏感现象 ${ }^{[19]}$. 具有缺口的 CS-PAM 微晶-共 价水凝胶双网络水凝胶 $\sigma_{\mathrm{f}} / E$ 值为 2.71 , 在变形的过程中 缺口增长缓慢, 表现出优异的缺口钝化行为(图 6C).

水凝胶在变形的过程中, 壳聚糖微晶网络会发生解 交联, 从而在应力应变曲线中产生滞后环, 滞后环的面 积代表了壳聚糖物理网络单位体积内耗散的能量. 当应 变为 $300 \%$ 时, 滞后环的面积占总功的 $86 \%$, 说明壳聚 糖网络的解交联行为是非常有效的能量耗散机制(图 $6 \mathrm{D})$. 可逆的壳聚糖微晶网络在破坏之后能够得以重建, 赋予微晶-共价双网络水凝胶优异的自恢复能力(图 6E) 和抗软化性能, 克服了传统水凝胶及双化学网络水凝胶 受力后软化、易疲劳的问题. 
A

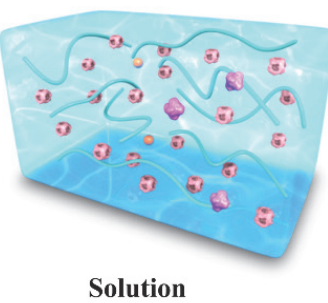

B

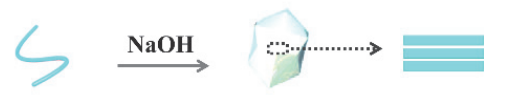

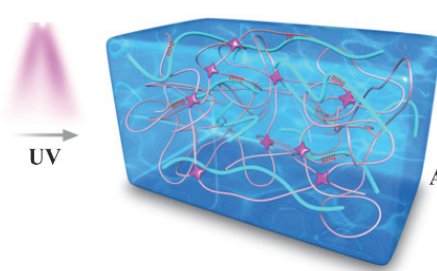

Composite Hydrogel

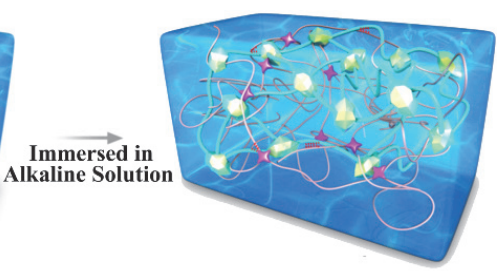

Microcrystalline-Covalent DN Hydrogel

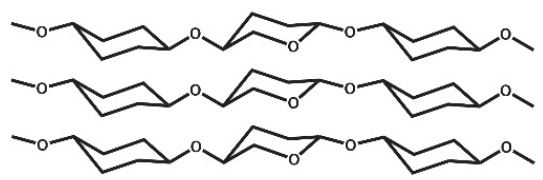

图 5 壳聚糖-聚丙烯酰胺微晶-共价双网络水凝胶的制备示意图 ${ }^{[12]}$

Figure 5 Scheme of fabrication of chitosan-polyacrylamide microcrystalline-covalent DN hydrogel ${ }^{[12]}$
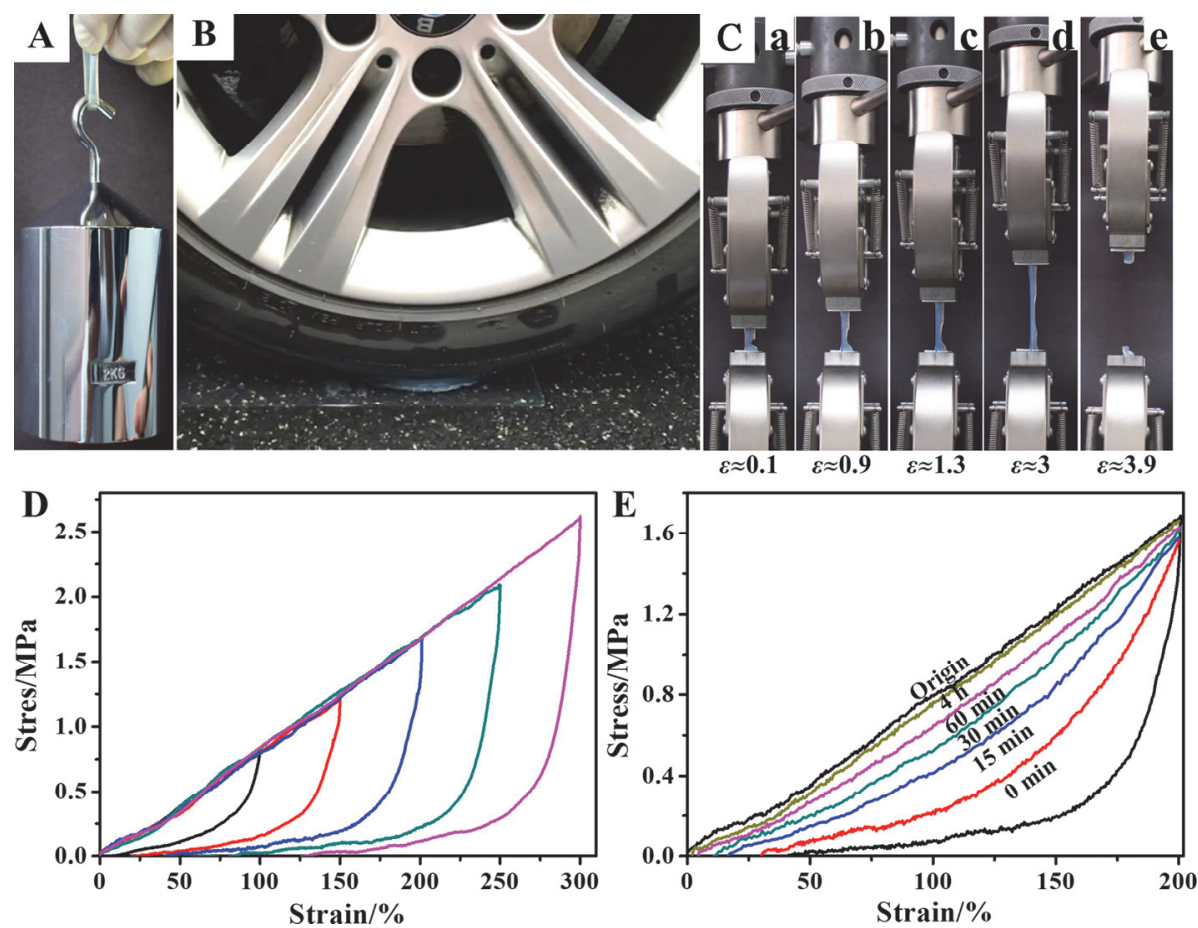

图 6 壳聚糖-聚丙烯酰胺微晶-共价双网络水凝胶优异的(A)负重能力、(B)抗碾压性、(C)缺口不敏感性、(D)能量耗散和(E)自恢复性能 ${ }^{[12]}$

Figure 6 Mechanical performance of chitosan-polyacrylamide microcrystalline-covalent DN hydrogel: (A) load-bearing capacity, (b) rolling resistance, (C) crack blunting, (D) energy dissipation and (E) self-recoverability ${ }^{[12]}$

\section{2 链缠结-共价双网络水凝胶}

将壳聚糖复合水凝胶浸泡于饱和的 $\mathrm{NaCl}$ 溶液中, 在强烈的盐析作用下, 壳聚糖分子间的静电排斥力减 小, 疏水作用力增强, 促使壳聚糖分子链移动并聚集, 聚集形成的缠结点作为壳聚糖网络的物理交联点 ${ }^{[9-11]}$, 从而形成壳聚糖-聚丙烯酰胺链缠结-共价双网络水凝胶 (图 7A). 与均匀致密的微晶-共价双网络水凝胶相比, 链缠结-共价双网络水凝胶孔径均匀性稍差, 综合力学 性能也相对稍弱.

在水中放置一段时间后, 壳聚糖链缠结网络会逐渐 解离, 宏观凝胶会转变为溶胶. 将链缠结-共价双网络 水凝胶放置在水中时, 其内部环境间的渗透压差会导致
凝胶吸收水分并且体积溶胀, 链缠结网络逐渐解离, 水 凝胶由不透明变化为透明状态(图 7B). 链缠结网络完全 瓦解后, 链缠结-共价双网络水凝胶完全转变为复合水 凝胶，表现出和复合水凝胶相同的平衡溶胀率(图 7C). 壳聚糖的微晶和链缠结网络在水中会遭到部分或者完 全的破坏, 因而微晶-共价和链缠结-共价水凝胶吸水溶 胀(平衡溶胀率分别为 140 和 45)后力学性能急剧下降, 因而需要构建一类更加稳定的壳聚糖物理网络, 从而制 备能够在水中抵抗溶胀, 保持高机械性能的壳聚糖基双 网络水凝胶.

\section{3 离子-共价双网络水凝胶}

壳聚糖分子链的 $D$-葡萄糖胺重复单元带有大量氨 
基，能够与带有负电荷的小分子盐(硫酸盐、磷酸盐或柠 檬酸盐)通过 “二叉” 或 “三叉” 配位键形成离子交联 网络 ${ }^{[11,16]}$. 我们使用低分子量、高渗透性的硫酸钠 $\left(\mathrm{Na}_{2} \mathrm{Sul}\right)$ 和高电荷密度的柠檬酸钠 $\left(\mathrm{Na}_{3} \mathrm{Cit}\right)$ 溶液用于交 联复合水凝胶来构建两种低溶胀的壳聚糖-聚丙烯酰胺 离子-共价双网络水凝胶(DN-Sul 和 DN-Cit) ${ }^{[20]}$, 其平衡 溶胀率分别为 2.1 和 0.8 (图 8). 与海藻酸钠离子网络相 比，壳聚糖离子网络具有两个优势：(1)网络中 “二叉”
或 “三叉” 配位键的密度大，因而网络的刚性更大，在 水中的稳定性也更好; (2)凝胶变形的过程中, 短链壳聚 糖网络更容易发生解离从而耗散能量, 增㓞水凝胶的效 果愈加显著. DN-Cit 水凝胶表现了非常优越的机械性 能: 拉伸强度为 $5.6 \mathrm{MPa}$, 弹性模量为 $1.3 \mathrm{MPa}$, 断裂韧 性为 $14.0 \mathrm{~kJ} \bullet \mathrm{m}^{-2}$, 优于已报道的大多数物理-化学和物 理-物理双网络水凝胶. 柠檬酸根与葡萄糖胺的 “三叉” 配位作用强，形成的离子网络致密，在水中的稳定性较

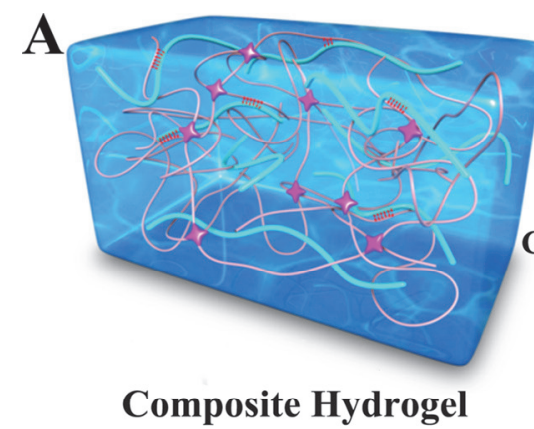

B

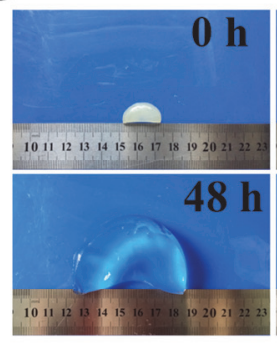

Immersed in Chloride Solution

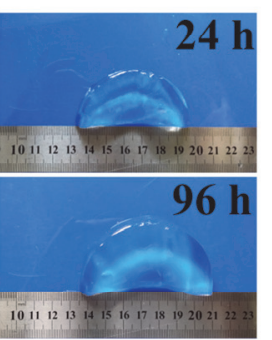

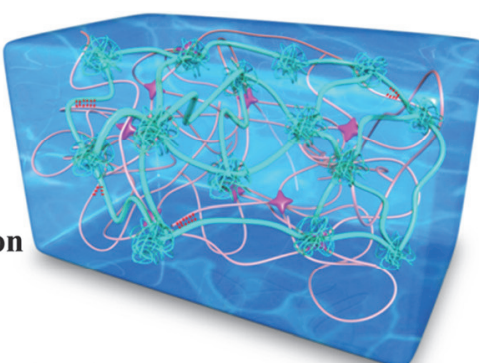

Chain-entanglent-Covalent DN Hydrogel

图 7 壳聚糖-聚丙烯酰胺链缠结-共价水凝胶的(A)制备示意图和(B, C) 溶胀行为 ${ }^{[12]}$

Figure 7 (A) Scheme of fabrication of chitosan-polyacrylamide chain-entanglement-covalent DN hydrogel and (B, C) its swelling behavior in water ${ }^{[12]}$

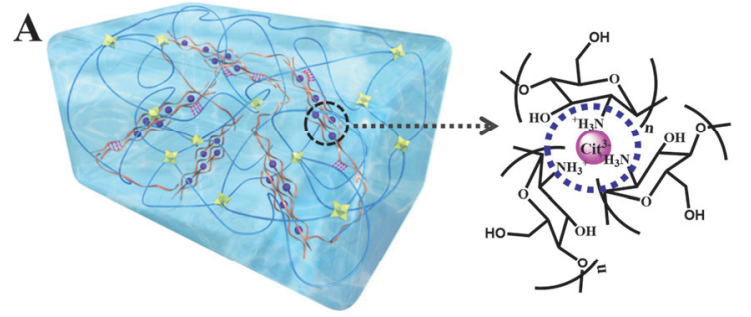

Ionic-Covalent DN Hydrogel

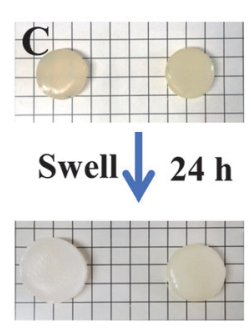

DN-Sul

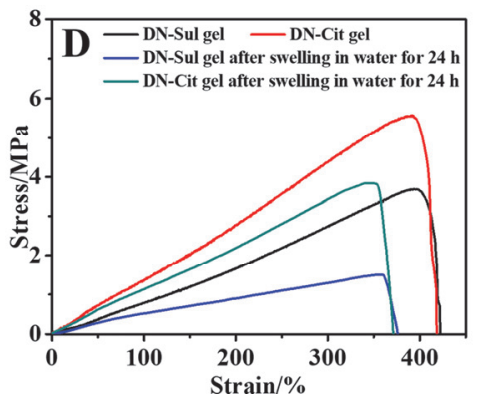

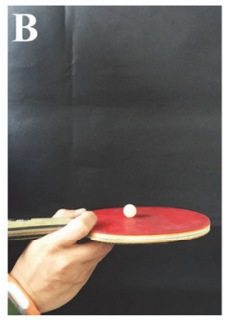
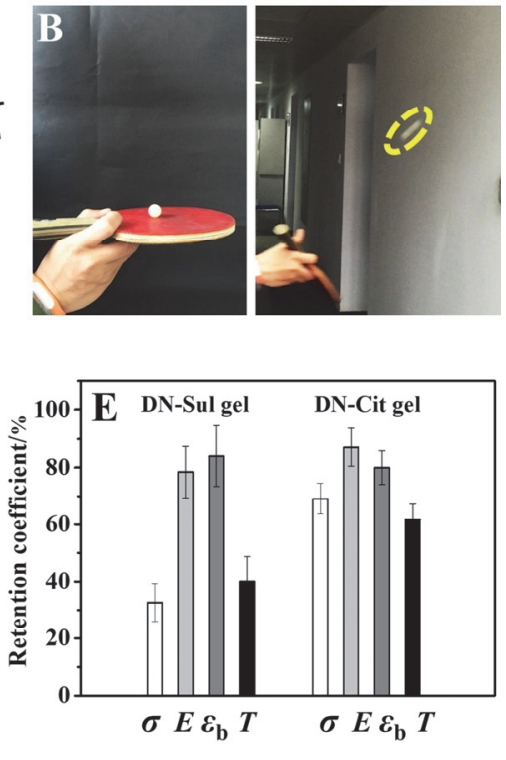

图 8 壳聚糖-聚丙烯酰胺离子-共价双网络水凝胶的(A)结构示意图、(B)高弹性、(C)溶胀行为及(D, E)溶胀前后的机械性能对比 ${ }^{[20]}$

Figure 8 (A) Structure and (B) high elasticity of chitosan-polyacrylamide ionic-covalent DN hydrogel. (C) Swelling behavior and (D, E) mechanical performance of the original and swollen hydrogel ${ }^{[20]}$ 
好, 因而 DN-Cit 水凝胶在水中溶胀 $24 \mathrm{~h}$ 后综合力学性 能依然能够保持原始性能的 $60 \%$ 以上. 壳聚糖-聚丙烯 酰胺离子-共价双网络水凝胶能够在一定程度上解决水 凝胶在水中溶胀后力学性能急剧下降的难题, 能够应用 于水下探测器、水下制动器等领域.

\section{4 普适性}

构建壳聚糖基双网络水凝胶的浸泡策略具有普适 性, 适用于各种化学网络或物理网络与壳聚糖相结合的 壳聚糖基复合水凝胶, 将其分别浸泡于碱性溶液、卤盐 溶液和多价阴离子盐溶液中能够得到壳聚糖的微晶、链 缠结和离子网络, 从而构建不同类型的高强韧双网络水 凝胶. 此外, 使用功能性分子的盐溶液来浸泡壳聚糖复 合水凝胶可以得到功能化的双网络水凝胶. 近几年来, 研究者们发展了壳聚糖-聚羟乙基丙烯酰胺离子-共价双 网络水凝胶 ${ }^{[21]}$ 、木质素-壳聚糖-聚乙烯醇离子-微晶双物 理网络水凝胶 ${ }^{[22]}$ 和壳聚糖-聚乙二醇二丙烯酸酯离子-共 价双网络水凝胶 ${ }^{[23]}$ 等多种高强韧水凝胶, 并探究了在 抗菌材料、伤口修复、药物释放和组织工程等领域的应 用. 此方法为双网络水凝胶的制备提供了一种新思路, 有利于拓展其在生物医药、仿生皮肤和柔性可拉伸器件 等领域的应用.

\section{4 结构与性能调控}

双网络水凝胶结构和性能的调控较为繁琐, 主要集 中在制备前期通过改变各成分的含量、交联剂的用量及 两个网络间的比例等来实现 ${ }^{[24]}$, 这意味着每一次调控 均需要重新制备一批新的凝胶, 将带来大量的实验操作 和时间精力的浪费. 第一网络的性质对于双网络水凝胶 的力学有重要的影响, 改变第一网络的含量、刚性或韧 性能够调控双网络水凝胶的机械性能 ${ }^{[25-26]}$. 因而我们考 虑是否可以先制备一种具有优异延展性的壳聚糖基复 合水凝胶, 然后采用不同的浸泡溶液类型和浸泡时间来
调控壳聚糖物理网络的类型、密度和刚性，从而实现对 水凝胶结构和机械性能的调控? 为了验证这一猜想，我 们将高延展性的壳聚糖-聚丙烯酰胺复合水凝胶浸泡于 柠檬酸钠溶液中，通过简单地调节浸泡时间来调控壳聚 糖离子网络的交联程度, 改变壳聚糖离子网络的密度和 刚性, 研究壳聚糖-聚丙烯酰胺离子-共价双网络水凝胶 机械性能的演变. 结果发现增加浸泡时间, 水凝胶的微 观结构更加致密, 刚性增强, 延展性变差, 韧性先增大 后降低. 因而简单地调控浸泡时间能够实现水凝胶的微 观结构和机械性能在较宽范围内灵活调控: 孔径大小 $0.1 \sim 3 \mu \mathrm{m}$, 弹性模量 $0.06 \sim 1.5 \mathrm{MPa}$, 拉伸强度 $0.1 \sim 5.6$

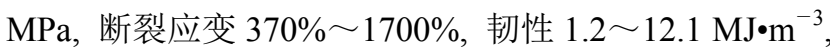
能够满足很多应用领域的机械需求(图 9). 同样, 改变浸 泡溶液的类型能够调整壳聚糖的网络类型、密度和刚性, 从而得到具有不同结构和机械性能的壳聚糖基双网络 水凝胶. 因而简单调整浸泡溶液种类和控制浸泡时间就 可以实现水凝胶的结构和性能的灵活调控, 能够促进双 网络水凝胶的发展与应用.

\section{5 应用}

壳聚糖基双网络水凝胶具有三个显著特点: (1)可逆 的壳聚糖物理网络能够为水凝胶提供有效的能量耗散 机制，提高水凝胶材料的机械性能(图 10A); (2)在电场 作用下，水凝胶中的自由离子可以迁移到相反方向，赋 予水凝胶良好的离子导电性(图 10B); (3)水凝胶中的离 子能够减少水分子的聚集，降低水分子之间的氢键，抑 制水形成冰晶，达到降低水凝胶凝固点的目的(图 10C). 壳聚糖基双网络水凝胶具有优异机械性能、导电性能和 抗冻性能，能够应用于柔性电子、抗冻敷料和可穿戴设 备等领域. 此外, 壳聚糖自身的抗菌、止血、促进伤口 愈合的特点也有利于壳聚糖基双网络水凝胶应用于生 物医用领域.

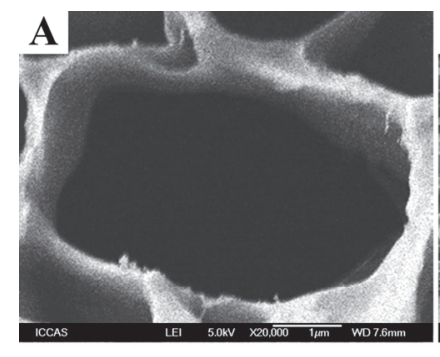

D

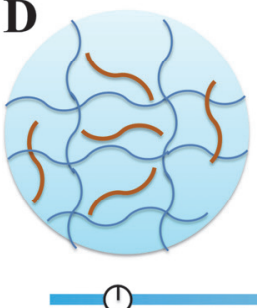

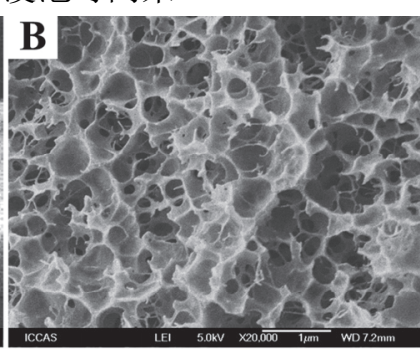
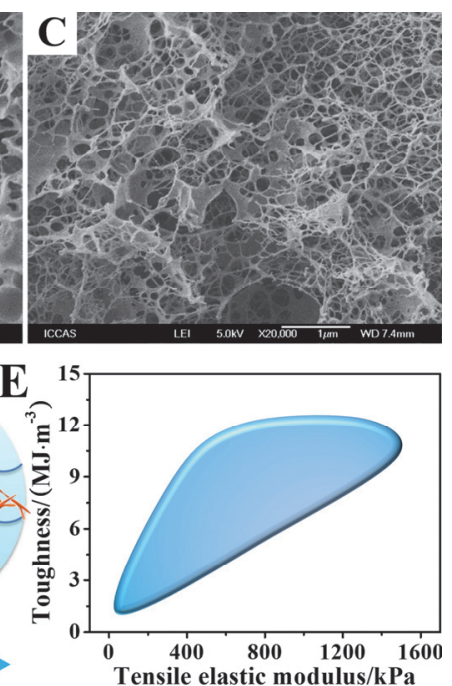

图 9 水凝胶结构和性能的灵活调控 ${ }^{[20]}$

Figure 9 Flexible adjustment of structure and mechanics of hydrogel ${ }^{[20]}$ 
A
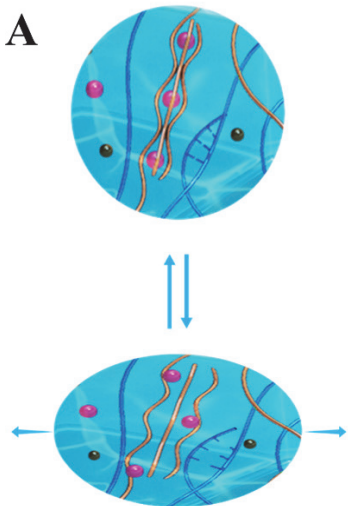

Reversible energy dissipation mechanism
B

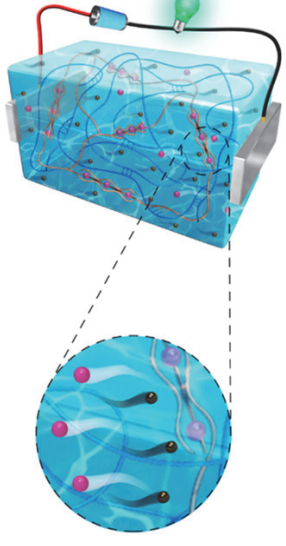

Ionic conductivity

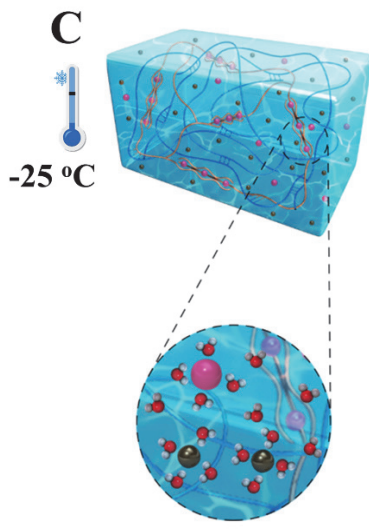

Low freezing point

图 10 高强韧导电抗冻的壳聚糖基双网络水凝胶的(A)能量耗散机制、(B)离子导电机理和(C)抗冻机理 ${ }^{[27]}$

Figure 10 (A) Energy dissipation mechanism, (B) ionic conductivity and (C) anti-freezing performance of high-mechanical chitosan-based DN hydro$\operatorname{gel}^{[27]}$

\section{1 抗冻敷料}

基于上述方法，我们制备了具有优异耐冻性能的壳 聚糖-聚羟乙基丙烯酰胺(CS-PHEAA)离子-氢键水凝胶, 水凝胶中的离子和 PHEAA 分子链上的极性基团共同赋 予了水凝胶优异的耐冻性能: (1) $\mathrm{Cit}^{3}{ }^{-}$和 $\mathrm{Na}^{+}$离子可以阻 碍水分子的聚集, 扰乱冰晶的形成; (2) PHEAA 链上的 亲水酰胺基团和稚基能够与水分子之间形成氢键, 减少 水分子间氢键作用力, 抑制冰晶的形成 ${ }^{[27]}$. 即便在 $-20{ }^{\circ} \mathrm{C}$ 环境下放置 $24 \mathrm{~h}$ 后, 水凝胶仍保持优异的柔软 性、可变形性和回弹性. 当温度降至 $-50{ }^{\circ} \mathrm{C}$ 时, 水凝胶 的断裂应变依然可达 $139 \%$.

具有低温耐受性的壳聚糖基双网络水凝胶能够作 为防冻材料, 避免皮肤在极端情况下冻伤. 如图 $11 \mathrm{~A}$ 所 示, 将在液氮中浸泡 $30 \mathrm{~min}$ 的硬币迅速贴附在小鼠背部 皮肤的三个区域, 分别为裸露的皮肤、复合水凝胶和双 网络水凝胶保护的皮肤. 当冷硬币撤去后, 双网络水凝 胶依然保持原始的湿软状态(图 11C). 皮肤组织学显微 镜图像结果表明, 裸露皮肤的角质层几乎完全脱离表皮 层, 真皮层出现大量的空洞; 复合水凝胶保护的皮肤出 现轻微的损伤, 而双网络水凝胶保护的皮肤表皮层和真 皮层都保持完整, 胶原纤维能够有序排列(图 11D $11 \mathrm{~F})$, 因而双网络水凝胶有望作为防冻涂层用于低温下 的皮肤保护.

\section{2 柔性传感器}

离子导电水凝胶在受到外界刺激时(如应变、压力), 水凝胶内部的离子通道和离子迁移路径发生变化, 水凝 胶的电学性质发生变化, 从而将外部刺激转化为可记录 的电信号. 壳聚糖基双网络水凝胶拥有优异的机械性能 和离子导电性, 能够组装形成电阻型电传感器或电容型 柔性传感器, 用于检测外界形变和应力的刺激 ${ }^{[27-28]}$. 以 壳聚糖-聚羟乙基丙烯酰胺双物理网络水凝胶为例, 水
凝胶的电阻在拉伸的过程中随拉伸应变的增加而增大, 在压缩过程中随应变或应力的增大而减小. 由于壳聚糖 基双网络水凝胶具有优异的弹性、超压缩性、可拉伸性 和抗疲劳性, 因而可组装成为多模式传感器同时检测应 变(拉伸、压缩和弯曲)和应力.

相对于其它离子型水凝胶传感器, 壳聚糖基双网络 水凝胶组装形成的传感器具有以下优势: (1)高灵敏性, 应变传感器常温下的灵敏系数为 6.6 (应变为 $400 \%$ ), 低 温下的灵敏系数为 3.9 (应变为 $280 \%$ ), 压力传感器常温 下的灵敏性为 $0.208 \mathrm{kPa}^{-1}$, 超过了目前大多数文献报道 的离子导电水凝胶的灵敏性 ${ }^{[28]}$; (2)优异的循环稳定性、 优异的抗疲劳性和机械稳定性保证传感器能够在 300 次 连续循环过程中保持灵敏的响应性和优异的循环稳定 性；(3)宽监测范围，压缩应变(高达 $80 \%$ )和压力(高达 $3.23 \mathrm{MPa}$ )的检测范围超过了目前大多数文献中报道的 水凝胶压力传感器的监测范围 ${ }^{[27]}$. 这种高灵敏、宽监测 范围、稳定的多模式传感器在柔性电子、人机交互、健 康诊疗、智能机器人等领域有巨大的应用潜力.

\section{3 可穿戴设备}

壳聚糖基双网络水凝胶对多种形变和压力均具有 灵敏的监测能力, 能够作为一种可穿戴传感设备实时监 测人体的活动. 如图 $12 \mathrm{~A} \sim 12 \mathrm{C}$ 所示, 水凝胶能够灵敏 地监测皱眉和吞咽等细微动作并识别人体关节的运动, 每一个动作都能产生清晰的电信号, 并且在循环过程中 相对电阻变化曲线呈现出稳定的响应峰谷. 此外, 水凝 胶可作为 “离子皮肤” 用于识别所承担压力的大小(图 12D). 水凝胶同样可以在雪天等低温环境中实时监测人 体的活动, 并且表现出良好的响应稳定性(图 12E). 因 此壳聚糖基双网络水凝胶能够作为可穿戴设备在宽温 域内对人体大小生理活动实现灵敏且稳定的监测, 为发 展可监测人体全范围运动的新型可穿戴柔性传感器提 

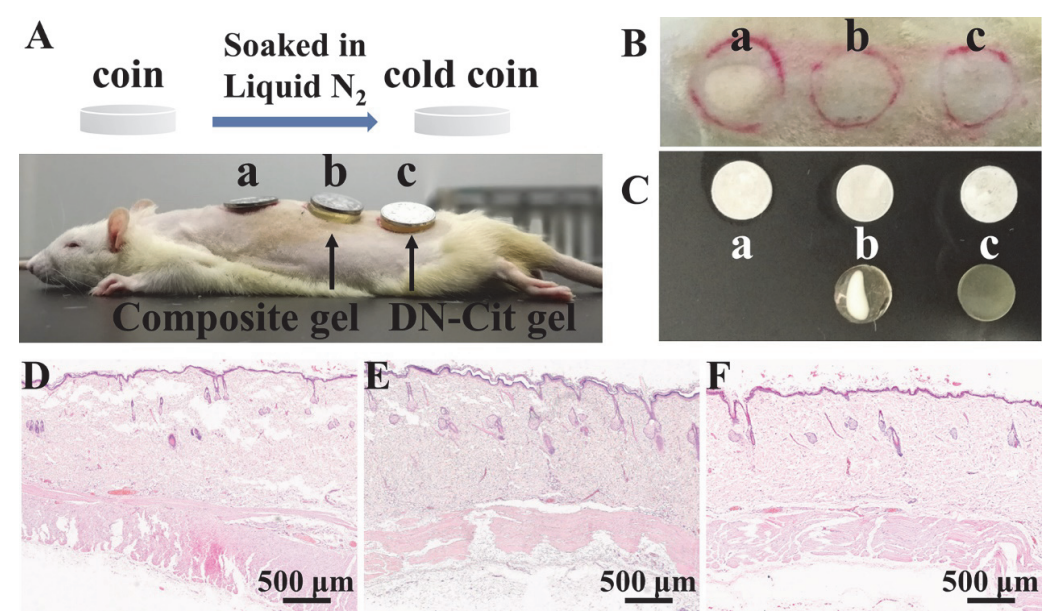

图 11 壳聚糖-聚羟乙基丙烯酰胺双网络水凝胶作为皮肤的防冻材料. (A)冻伤模型示意图; 移除冷硬币之后的(B)皮肤、(C)硬币(上)及水凝胶(下) 的照片; 皮肤组织学显微镜图像: (D)未被保护的皮肤、(E)被复合水凝胶和(F)被双网络水凝胶保护的皮肤 ${ }^{[27]}$

Figure 11 CS-PHEAA DN hydrogel as ant-freezing dressing to protect skin from frostbite. (A) Schematic diagram of frostbite model. Photos of (B) skin, (C) cold coins (top) and hydrogels (bottom) after coving the cold coin for $30 \mathrm{~s}$. Histological microscopy images of (D) bare skin, (E) skin protected by composite hydrogel and (F) skin protected by DN hydrogel ${ }^{[27]}$
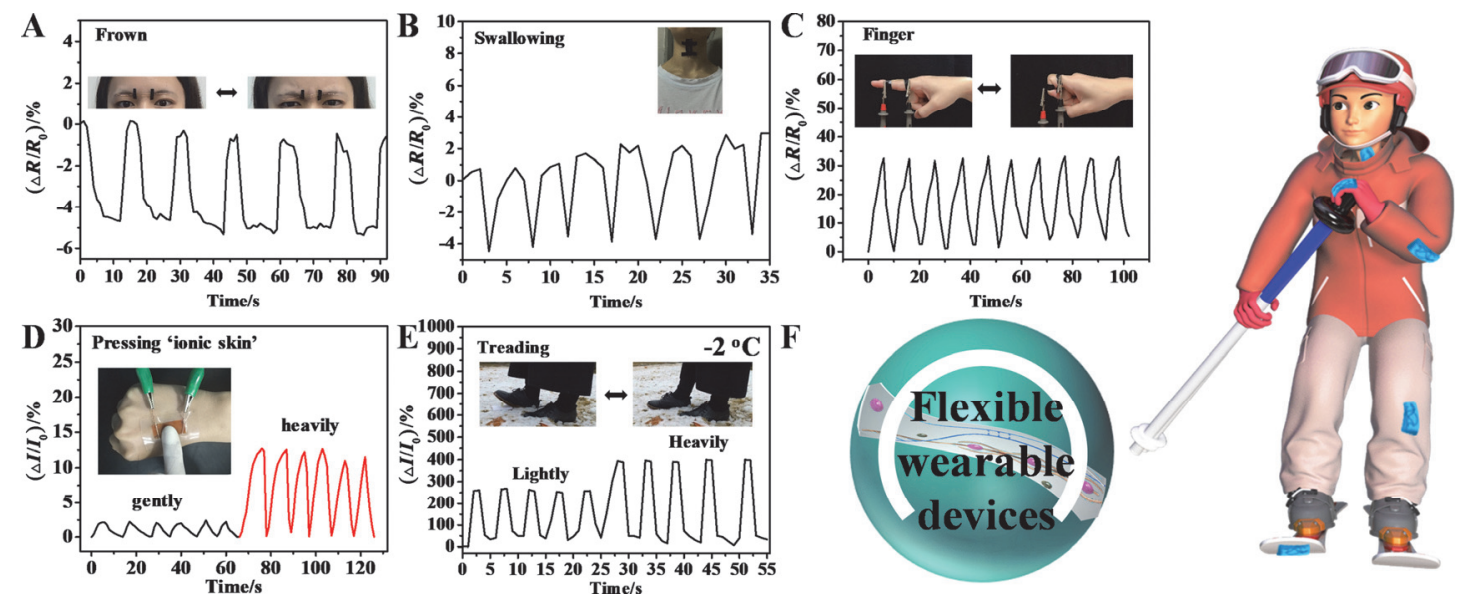

图 12 壳聚糖基双网络水凝胶作为多模式抗冻可穿戴设备检测人体活动 ${ }^{[27-28]}$

Figure 12 Chitosan-based DN hydrogel serving as anti-freezing and multi-model wearable device to detect various human motions ${ }^{[27-28]}$

供了可能.

\section{4 生物医用材料}

壳聚糖具有抗菌、止血、促进伤口愈合的优势, 在 开发伤口敷料等方面具有显著的优势 ${ }^{[29-30]}$. 高机械性能 的壳聚糖基双网络水凝胶能够避免对伤口的二次伤害 和污染, 能够快速激活凝血系统、减少出血、杀灭细菌, 有效促进细胞增殖和迁移, 加速创面愈合. 周等 ${ }^{[23]}$ 报道 了一种木质素-壳聚糖-聚乙烯醇离子-微晶双物理网络 水凝胶, 不仅能够有效地穿透金黄色葡萄球菌的细胞膜 以达到杀菌效果, 而且可以维持伤口周围潮湿的愈合环 境, 促进再上皮化, 从而使得结缔组织得到快速重塑和 排列, 达到促进伤口愈合的效果. 卢等 ${ }^{[22]}$ 发展了高粘 附、高机械性能和细胞相容性的壳聚糖-聚乙二醇二丙 烯酸酯离子-共价双网络水凝胶, 并负载广谱抗菌活性 的多肽和促血管生成素表达的质粒两种活性物质, 在全 层皮肤修复中有效抑制感染, 形成微血管, 从而协同促
进伤口愈合.

\section{6 总结与展望}

本工作简要总结了我们课题组用于构建壳聚糖基 双网络水凝胶的策略, 开发出三种刚而脆的壳聚糖物理 网络, 分别为微晶网络、链缠结网络和离子网络, 并将 其作为第一网络成功地构建了多种高强㓞导电抗冻的 双网络水凝胶. 水凝胶具有优异的机械性能、离子导电 性和抗冻结性能，结构和性能可以通过改变浸泡溶液的 类型和浸泡时间进行灵活快速的调控. 构建壳聚糖基双 网络水凝胶的策略简单通用, 第二网络的类型不受限 制, 能够制备具有功能性的高强韧水凝胶. 在此基础上, 我们探索了水凝胶在抗冻敷料、柔性电子和可穿戴设备 领域的潜在应用价值. 这些研究极大丰富了高机械性能 水凝胶的种类, 为水凝胶的发展和应用开辟了新的道 路. 
尽管壳聚糖基双网络水凝胶在近些年取得了重要 的进展，但是目前所制备的水凝胶依然存在使用过程中 失水、表面粘合性差、自愈合能力欠缺等劣势，降低了 其实际应用价值. 因此, 我们希望未来有更多智能化的 壳聚糖基高强韧水凝胶被研究者们设计并开发出来, 进 一步提高其应用价值并拓展到更多的应用领域. 总而言 之, 这种制备简单、结构和性能可调、兼具高机械性能、 导电性和抗冻性的壳聚糖基双网络水凝胶将会在柔性 电子、可穿戴设备、智能机器、人机交互和组织工程领 域的研究和应用中拥有一席之地.

\section{作者简介}

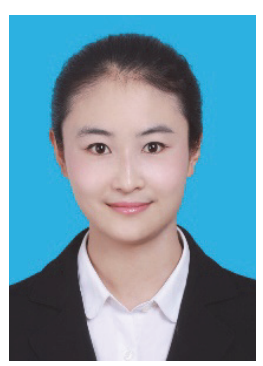

杨艳宇, 2017 年博士毕业于中国科学院化学研究所, 高分 子化学与物理专业, 导师为吴德成研究员, 现为郑州大学副 教授, 研究方向为高机械性能水凝胶材料的构筑与功能化研 究.

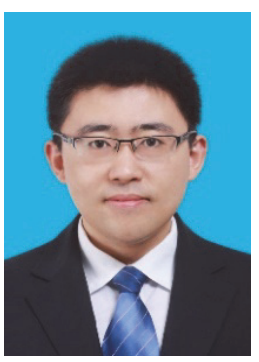

王星, 中国科学院化学研究所副研究员, 2014 年于中国科 学院化学研究所取得博士学位, 研究方向为医用高分子水凝 胶的可控制备、功能构筑及应用研究.

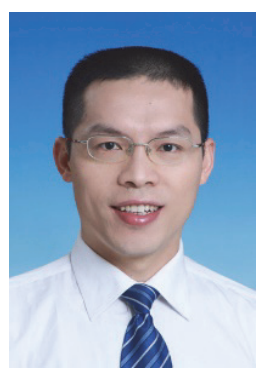

吴德成, 中国科学院化学研究所研究员、南方科技大学生 物医学工程系讲席教授. 研究方向为生物医用高分子、医用敷 料/耗材、生物成像、药物输运和组织工程.

\section{References}

[1] Gong, J. P. Science 2014, 344, 161.

[2] Chen, Q.; Zhu, L.; Zhao, C.; Wang, Q. M.; Zheng, J. Adv. Mater. 2013, 25, 4171 .

[3] Sun, J. Y.; Zhao, X. H.; Illeperuma, W. R. K.; Chaudhuri, O.; Oh, K. H.; Mooney, D. J.; Vlassak, J. J.; Suo, Z. G. Nature 2012, 489, 133.

[4] Stevens, L.; Calvert, P.; Wallace, G. G.; Panhuis, M. I. H. Soft Matter 2013, 9, 3009.

[5] Bakarich, S. E.; Pidcock, G. C.; Balding, P.; Stevens, L.; Calvert, P.; Panhuis, M. I. H. Soft Matter 2012, 8, 9985.

[6] Yuan, N. X.; Xu, L.; Wang, H. L.; Fu, Y. P.; Zhang, Z.; Liu, L.; Wang, C. L.; Zhao, J. H.; Rong, J. H. ACS Appl. Mater. Interfaces 2016, 8, 34034.

[7] Chen, Q.; Zhu, L.; Chen, H.; Yan, H. L.; Huang, L. N.; Yang, J.; Zheng, J. Adv. Funct. Mater. 2015, 25, 1598.

[8] Li, J. Y.; Suo, Z. G.; Vlassak, J. J. J. Mater. Chem. B 2014, 2, 6708.

[9] Ladet, S.; David, L.; Domard, A. Nature 2008, 452, 76.

[10] Porath, J.; Sundberg, L.; Fornsted, N.; Olsson, I. Nature 1973, 245, 465.

[11] Bhattarai, N.; Gunn, J.; Zhang, M. Q. Adv. Drug Delivery Rev. 2010, 62,83 .

[12] Yang, Y. Y.; Wang, X.; Yang, F.; Shen, H.; Wu, D. C. Adv. Mater. 2016, 28, 7178

[13] Dash, M.; Chiellini, F.; Ottenbrite, R. M.; Chiellini, E. Prog. Polym. Sci. 2011, 36, 981.

[14] Zhang, Y. L.; Yang, B.; Xu, L. X.; Zhang, X. Y.; Tao, L.; Wei, Y. Acta Chim. Sinica 2013, 71, 485 (in Chinese). (张亚玲, 杨斌, 许亮 金金, 张小勇, 陶磊, 危岩, 化学学报, 2013, 71, 485.)

[15] Xiong, Y.; Yan, K.; Bentley, W. E.; Deng, H. B.; Du, Y. M.; Payne, G. F.; Shi, X. W. ACS Appl. Mater. Interfaces 2014, 6, 2948.

[16] Shu, X. Z.; Zhu, K. J. Int. J. Pharm. 2002, 233, 217.

[17] Shu, X. Z.; Zhu, K. J.; Song, W. H. Int. J. Pharm. 2001, 212, 19.

[18] Guan, J.; Xu, H. X.; Huang, Y. F.; Tian, K.; Shao, Z. Z.; Chen, X. Acta Chim. Sinica 2010, 68, 89 (in Chinese). (管娟, 许惠心, 黄郁 芳, 田琨, 邵正中, 陈新, 化学学报, 2010, 68, 89.)

[19] Luo, F.; Sun, T. L.; Nakajima, T.; Kurokawa, T.; Zhao, Y.; Bin Ihsan, A.; Guo, H. L.; Li, X. F.; Gong, J. P. Macromolecules 2014, 47, 6037.

[20] Yang, Y. Y.; Wang, X.; Yang, F.; Wang, L. N.; Wu, D. C. Adv. Mater 2018, 30, 1707071.

[21] Zhang, J.; Chen, L.; Shen, B.; Chen, L.; Feng, J. Carbohydr. Polym. 2019, 225, 115160 .

[22] Huang, L.; Zhu, Z.; Wu, D.; Gan, W.; Zhu, S.; Li, W.; Tian, J.; Li, L.; Zhou, C.; Lu, L. Carbohydr. Polym. 2019, 225, 115110.

[23] Zhang, Y.; Jiang, M.; Zhang, Y.; Cao, Q.; Wang, X.; Han, Y.; Sun, G.; Li, Y.; Zhou, J. Mater. Sci. Eng. C 2019, 104, 110002.

[24] Li, J. Y.; Illeperuma, W. B. K.; Suo, Z. G.; Vlassak, J. J. ACS Macro Lett. 2014, 3, 520.

[25] Chen, Q.; Wei, D. D.; Chen, H.; Zhu, L.; Jiao, C. C.; Liu, G.; Huang, L. N.; Yang, J.; Wang, L. B.; Zheng, J. Macromolecules 2015, 48, 8003

[26] Xin, H.; Saricilar, S. Z.; Brown, H. R.; Whitten, P. G.; Spinks, G. M. Macromolecules 2013, 46, 8393.

[27] Yang, Y. Y.; Yang, Y. T.; Cao, Y. X.; Wang, X.; Chen, Y. R.; Liu, H. Y.; Gao, Y. F.; Wang, J. F.; Liu, C.; Wang, W. J.; Yu, J. K.; Wu, D. C. Chem. Eng. J. 2021, 403, 126431.

[28] Liu, H. Y.; Wang, X.; Cao, Y. X.; Yang, Y. Y.; Yang, Y. T.; Gao, Y. F.; Ma, G. S.; Wang, J. F.; Wang, W. J.; Wu, D. C. ACS Appl. Mater. Interfaces 2020, 12, 25334.

[29] Qu, J.; Zhao, X.; Liang, Y.; Zhang, T.; Ma, P. X.; Guo, B. Biomaterials 2018, 183, 185.

[30] Chedly, J.; Soares, S.; Montembault, A.; von Boxberg, Y.; Veron-Ravaille, M.; Mouffle, C.; Benassy, M. N.; Taxi, J.; David, L.; Nothias, F. Biomaterials 2017, 138, 91. 